the alternatives posed by Dr O'Leary can be shown to be at least as successful?

The Young People's Unit

P. G. Wells

Macclesfield SK10 3JS

\section{Asylum, asylums and rehabilitation}

DEAR SIRS

Several speakers at the rehabilitation session of the Royal College's 1989 Annual Meeting and, a few days earlier, at the Netherne Annual Rehabilitation Study Day, discussed the challenging problems of long-term severely disabled patients in the era of care in the community. Unfortunately, the debate tended to confuse two separate, though interrelated issues: the need to provide long-term shelter and support (asylum) and the closure of large mental hospitals (asylums). Numerous contributors to the Bulletin, notably Abrahamson (1988) and Garelick (1988), have cogently clarified the argument for the provision of asylum as part of psychiatric services; this is a view shared by many concerned psychiatrists, and not that of reactionaries who cling to an outmoded ideology of institutional care. Nevertheless, there is little evidence that the mental hospitals' role of providing asylum to the group of severely disabled patients who need it is being effectively translated into community settings. The rapid run down of the hospitals has dictated the extent and pace of discharging such patients to poorly planned, inadequately resourced community environments, as pointed out in the statement by the Coordinators of National Demonstration Services in Rehabilitation (1987). Many of these patients do not receive the continued care, shelter and support inherent in good rehabilitation practice; homelessness, destitution, involvement with the police and lack of basic medical care are prevalent.

The reassuring statement by Mr Roger Freeman, Parliamentary Under Secretary of State for Health, which was issued to Health Authorities (15 June 1989), makes the point that the White Paper policy for the "development of locally based hospital and community services, including facilities providing long-term asylum for those who need it, and as a consequence the closure of very large mental hospitals, has remained essentially unchanged since, supported by successive Governments" and that hospitals should only close "when proper alternative locally based services are available". It has been evident, however, that there is often a gap between policy and implementation. It is for this reason that CONCERN (Care of the Neglected: Collaborative Education, Rehabilitation and Nursing) was formed, with the initiative of Dr Malcolm Weller (Lowry, 1989). CONCERN will act as a professional pressure group whose main aim is to encourage the slowing down of hospital closures so that the needs of severely disabled patients can be accurately demonstrated and until adequate community services are provided to meet those needs.

MoUNIR EKDAWI

Netherne Hospital

Coulsdon, Surrey CR3 IYE

\section{References}

ABrahamson, D. (1988) The need for asylum in society for the mentally ill or infirm. Bulletin of the Royal College of Psychiatrists, 12, 76-77.

EkDAwI, M. Y. et al (1987) National Demonstration Services-Statement on psychiatric rehabilitation and care in the community. Bulletin of the Royal College of Psychiatrists, 11, 207-209.

GARELICK, A. I. (1988) The decision to close an area mental hospital. Bulletin of the Royal College of Psychiatrists, 12 , 52-53.

LOWRY, S. (1989) Concern for discharging mentally ill patients. British Medical Journal, 298, 209-210.

\section{Psychiatric appraisal for the Mental Health Review Tribunal - an algorithm}

\section{DeAr Sirs}

At Mental Health Review Tribunals (MRHTs) a number of issues can arise which have general application to a majority of the patients seen. It is useful, therefore, for the medical member of a MHRT to cover the salient points likely to be relevant to the Tribunal at the pre-hearing psychiatric examination of the patient. For this purpose a set format, or algorithm, provides a structured record. This can be kept simple enough to be completed in a few minutes before the Tribunal and copies can be filled in or photocopied for the President and lay member of the MHRT. This approach might also be of help to Responsible Medical Officers (RMOs) who can assist MHRTs and possibly save themselves time and questions by presenting background information and details which the Tribunal will find of practical value in reaching a decision.

The following is a form of report which can be typed on to one side of standard A4 paper. Most of it is completed by just underlining the appropriate words applicable to the case.

D. A. SPENCER

Meanwood Park Hospital

Leeds LS6 4QD

\section{Reference}

Mental Health Review Tribunals in England \& Wales - A Guide for Members, 1988. 